\title{
Temperature dependence on the photovoltaic properties of selected thin-film modules
}

\author{
V. Perraki, G. Tsolkas
}

Department of Electrical and Computer Engineering, University of Patras, 26110 Patras, Greece

Email address:

perraki@ece.upatras.gr(V. Perraki), tsolkas.george@gmail.com(G. Tsolkas)

To cite this article:

V. Perraki, G. Tsolkas. Temperature Dependence on the Photovoltaic Properties of Selected Thin-Film Modules. International Journal of Renewable and Sustainable Energy. Vol. 2, No. 4, 2013, pp. 140-146. doi: 10.11648/j.ijrse.20130204.12

\begin{abstract}
An important requirement in the employment of the different existing PV technologies is the understanding of the performance exhibited by each technology, once installed outdoors. Such records are necessary since the outdoor PV electrical characteristics are different from those corresponding to STC (which rarely occurs outdoors) information listed in manufacturer data-sheets. Therefore the PV monitoring and evaluations, under different environmental conditions, are indispensable for the architects and PV systems installers, in order to accurately size the installations. In this paper the influence of temperature on the photovoltaic parameters of amorphous silicon (a-Si) and copper indium diselenide (CIS) thin film modules has been investigated, as well as the energy produced under actual operating conditions. The current-voltage characteristics and maximum power have been recorded at regular intervals, for one year in the Mediterranean climate city of Patras, Greece (latitude $38^{\circ}$ ). Patras averages over 4.2 peak sun hours (PSH) per day and module working temperatures between $16{ }^{\circ} \mathrm{C}$ and $60^{\circ} \mathrm{C}$. Our results have shown that, the percentage reduction of the open circuit voltage with temperature increase is greater for the CIS than for the a-Si modules. The short circuit current temperature coefficient for the CIS modules is positive at low and medium temperatures, though over the entire range of working temperature remains approximately constant with a slight tendency to reduce. The maximum power decreases almost linearly, while the efficiency for temperatures higher than $50^{\circ} \mathrm{C}$ reduces sharply. It is remarkable that with respect to the temperature increase the a-Si modules efficiency remains very near to the rated value, and the short circuit current temperature coefficient and the power coefficient are positive. The fill factor for these modules decreases linearly and equally as a function of temperature. The series and parallel resistance for the a-Si decrease slightly with temperature increase, whereas for the CIS the series resistance increases and the parallel resistance decreases in a more pronounced way. Maximum year-round energy production corresponds to the tilt angles of about 20 and 50 degrees in the summer and winter respectively.
\end{abstract}

Keywords: Thin Film, Module, A-Si, CIS, Temperature Coefficient, Tilt Angle

\section{Introduction}

When designing any solar system there is an essential requirement to accurately estimate the output from the proposed PV array under operating conditions. An appropriately sized PV array enables clients, to receive at reasonable cost a reliable energy supply. It is however known that PV modules of dissimilar technologies have different seasonal performances, due to the variations in the efficiency, in the different temperature coefficients of the module parameters and in the additional result of degradation. PV modules are rated at standard test conditions (STC) that is, $1 \mathrm{~kW} / \mathrm{m}^{2}$ irradiance, $25{ }^{0} \mathrm{C}$ module temperature, AM 1.5 solar spectrums and zero angle of incidence. Although this information is useful at comparing PV modules perfor- mance at rating conditions, it does not represent what is typically experienced under outdoor operation. Discrepancies between the ranking and evaluating conditions are assigned to the large annual variations that are practiced in incident angle, solar spectrum, operating temperature and solar irradiance as compared to the STC conditions. Operating temperature is a crucial parameter that has great influence in the behavior of a PV system, as it modifies system efficiency and energy yield. It depends on the module encapsulating material, the thermal dissipation, the absorption properties, the functioning point of the module, as well as the irradiance intensity, ambient temperature, wind speed and the accurate installation status [1]. It is thus important to perform a quantitative analysis of the temperature influence on PV module properties such as voltage, 
current, power, and efficiency under real operation conditions [2]. Moreover, practical installation optimization could be achieved by using hybrid photovoltaic thermal (PVT) modules and / or PV systems one/two axes trackers PV systems.

It has been reported that modules of different technologies cannot be adapted to certain specific climates in the same way. a-Si solar modules present a decrease of conversion efficiency at the original irradiation [3] and it is significant that the durability and the outdoor performance of the modules should be tested. On the other hand this type of modules may be more adjusted to tropical climates [4] [5]. a-Si modules display a better performance when the ambient and consequently the working temperatures of the modules are high [6]. In such an environment with high ambient temperatures and high humidity during the wet season, an amorphous silicon array may generate up to $20 \%$ more energy than a Poly-crystalline silicon (p-Si) array [7]. Amorphous silicon cells as well as compound CIS cells have shown great potential to move closer to commercialization. They require very little semiconductor material, achieving lower costs without, in the same time, sacrificing too much of the efficiency of photovoltaic devices. It is thus essential for the manufacturers to obtain information on how much energy these thin film PV modules produce under different circumstances and guarantee the reliability of the modules.

We have already published [8], the results of operation outdoors for a long time, for four different PV technologies.

The main results, had focused on the efficiency, energy yield and power ratio and there is no reason to be reproduced here, except to notice that these technologies have shown enormous potential in countries with high solar resource.

This paper presents the results of extensive outdoor testing of a-Si and CIS modules under varying meteorological conditions, by the use of our installation on the roof of the building of the department of Electrical and Computer Engineers at the University of Patras, Greece (latitude $38^{0}$ ).

Field test records were collected and analyzed focusing on the influence of temperature over the photovoltaic properties of these modules. The temperature coefficients have been calculated as well as the annual energy yield as a function of the modules tilt angle, in order to provide helpful information to PV system architects and installers.

\section{Effect of Temperature on the I-V Characteristics of PV Modules}

Temperature is the second most important factor (besides irradiance) affecting the performance of PV cells/modules as it has a major impact on the performance and degradation, particularly in countries with high temperatures. One of the simplest cells models is presented in figure 1 .

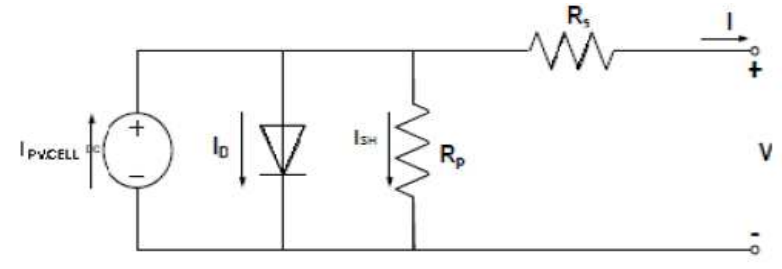

Figure 1. The electrical equivalent circuit of a solar cell [Muhammad Towhidur Rahman et al

Temperature affects directly, the exponential term, and indirectly all the other terms of the current-voltage equation of a solar cell/module (equation 1 ).

$$
I=I_{p h}-I_{0}\left[\exp \frac{q\left(V+R_{s} I\right)}{A k_{b} T}-1\right]-\frac{V+R_{s} I}{R_{p}}
$$

Where, $I_{p h}$ and $I_{0}$ stand for the photogenerated and the dark generated current respectively, $k_{b}$ for the Boltzmann's constant, $V$ for the voltage, and $T$ for the temperature. $R_{s}$ and $R_{p}$ stand for the series and the shunt resistance respectively, which are expressed by the relations 2 and 3 .

$$
R_{s}=-\frac{d V}{d I_{V o c}}-\frac{1}{I_{0}\left(q / n k_{b} T\right) e^{q V / n k_{b} T}}
$$

and

$$
R_{p}=V_{m p}+\frac{R_{s} I_{m p}}{I_{m p}-I_{p h}+I_{0}\left(e^{q\left(V_{m p}+R_{s} I_{m p}\right) / n k_{b} T}-1\right)}
$$

$I_{m p}, \mathrm{~V}_{m p}$ stand for the current and voltage at MPP. Due to their dissimilar nature these currents have different temperature dependencies. $I_{L}$, increases slightly with increasing temperature yielding to $0.09 \%$ for amorphous silicon, while $I_{0}$ which is related to the thermally generated carriers in the cell, increases exponentially with temperature. Only the magnitude of the exponential term reduces. The net effect is the reduction of the open-circuit voltage $V_{O C}$ linearly with increasing temperature. The magnitude of this reduction is inversely proportional to $V_{O C}$ that is cells with higher values of $V_{O C}$ undergo smaller reductions in voltage with increasing temperature. By the way of comparison, the rate for amorphous silicon solar cells extends from $-0.20 \% /{ }^{\circ} \mathrm{C}$ to $-0.30 \% /{ }^{\circ} \mathrm{C}$, depending on the processes the cell is made, which is lower than that of crystalline silicon cells. The overall effect of temperature on cell efficiency can be computed using these factors in combination with the characteristic equation. However, since the change in voltage is much stronger than the change in current, [1] the overall effect on efficiency tends to be similar to that on voltage. In fact most amorphous cells decline by $0.15-0.25 \% /{ }^{\circ} \mathrm{C}$.

Temperature changes affect the bandgap of the semiconducting material, and the series resistance $R_{S}$ of the module, depending on the photovoltaic material in question. The 
module temperature in the field depends on the irradiation, the ambient temperature, and the wind speed. For most technologies, a low module temperature is favorable, since it usually gives better results. Studying the module performance it is essential that you can assess results obtained at different temperatures, and calculate how much energy the module produces.

For this purpose, the temperature coefficient of each one of the module parameter X (short circuit current Isc, open circuit voltage Voc, power $P$, fill factor FF or efficiency $\eta$ ) is useful to be identified $[9,10]$. This coefficient has usually been defined at a standard temperature $\mathrm{T}_{\text {STC }}$, (a value of $25^{\circ} \mathrm{C}$ frequently used), however it depends on the temperature and the irradiance as well through the dependence of $X$ 's parameter. The behavior of the module's electrical parameters as a function of temperature has been found to be close to linear for a wide range of temperatures, consequently good approximations for the values of $\mathrm{X}(\mathrm{T})$ can be found with the relationship (4):

$$
X(T)=X_{S T C}+\alpha_{X}\left(T_{C}-T_{S T C}\right)
$$

Where $X(T)$ represents the value of $X$ parameter at a temperature $\mathrm{T}, \mathrm{X}_{\mathrm{STC}}$ its value at $\mathrm{T}_{\mathrm{STC}}, \mathrm{T}_{\mathrm{c}}$ the cell temperature usually set in Celsius degrees and $\alpha_{X}$ the temperature coefficient of each one of the module parameter $\mathrm{X}$ which can be expressed $[10,11]$ by the relation (5):

$$
\alpha_{X}=\left.\frac{1}{X} \frac{d X}{d T_{c}}\right|_{T_{S T C}}
$$

In this way, comparisons can be made between parameter values measured at different temperatures and different irradiance levels. This facilitates an analysis of how the module is performing even with the fluctuations in temperature and irradiance.

\section{Experimental Details}

A proper orientation and tilt angle of a module can maximize its energy production potential. For best year-round performance in most locations fixed arrays should be oriented to true South (North hemisphere). In case there is no possibility to move the surface at all, the optimal tilt angle for the maximum amount of direct irradiation is set out to the site's latitude. Tilting the surface up, however, causes the diffuse light portion to decrease. In regions where most of the irradiance occurs in summer, it may be beneficial to adjust the tilt angle for winter and summer. In Mediterranean sites however seasonal differences are less pronounced, making a seasonal tilt less critical.

In this study the outdoor facility with a data acquisition system has been installed on the roof of the building of the department of Electrical and Computer Engineers at the University of Patras, Greece (latitude $38^{0}$ ). An open rack is used in order to place the modules and the necessary instrumentation to record the parameters examined. Irradiance, ambient temperature, and module temperature have been recorded moreover for the purpose of the study.

The PV modules under investigation are commercially available, a- Si, and CIS modules with peak power 32 and $75 \mathrm{~W}$ respectively and they were installed in both fixed tilt and manual tracking modes. The experimental setup is composed of a pyranometer for the measurement of irradiance at the level surface of module, temperature sensors and a PVPM measuring device, for plotting the electric values of the $\mathrm{I}-\mathrm{V}$ and $\mathrm{P}-\mathrm{V}$ characteristic pairs. A personal computer is also interfaced to the above measuring procedure. The signals from PV modules are transmitted to the PVPM device which visualizes the data measured after each test. This system automatically measures meteorological data and operating condition data at an interval of some seconds and test results are stored in a database and can immediately be printed out in the form of a report containing all relevant data.

In order to achieve comparable results, the modules were installed under the same conditions and the experimental measurements were performed simultaneously, under various conditions of radiation and temperature and for several tilt angles.

\section{Results}

The SCHOTT Solar type ASI-F 32/12 a-Si modules and the thin film SHELL ECLIPSE 75-C CIS modules tested in this work have specifications according to the manufacturers which are reported in table 1.

All tests were performed, at the University of Patras' test facilities, representing a typical site across Greek midland with irradiation $4.2 \mathrm{KWh}$ /day $(\mathrm{PSH})$ yearly and mean annual outdoor temperature $17.7{ }^{\circ} \mathrm{C}$ [12]. The PV modules operated under clear and cloudy sky conditions in order to simulate yearly meteorological conditions in an efficient way.

The data obtained have been used to compute the temperature coefficients for $\mathrm{I}_{\mathrm{sc}}, \mathrm{V}_{\mathrm{oc}}, \mathrm{FF}, \mathrm{P}_{\max }$, and $\eta$ for a test period of one year. It is actually difficult to explore the impact of the temperature only, on $\mathrm{I}_{\mathrm{sc}}$ and $\mathrm{V}_{\mathrm{oc}}$ when a PV module is installed in a real environment, as all localized climatic parameters act simultaneously and its response represents the combined effect of these parameters. To achieve comparable results, the recorded data have been adjusted to the STC or corresponded to, an average solar irradiance of $980 \pm 2.1 \% \mathrm{~W} \cdot \mathrm{m}^{-2}$ and the same tilt angle. The modules were tested in a wide range of temperatures during the period of tests. Specifically the averaged operating temperatures of the modules were in the range of $20-55{ }^{\circ} \mathrm{C}$.

Fig. 2 shows the plots of the short circuit current $\mathrm{I}_{\mathrm{sc}}$ for the modules under investigation, versus the operating temperature. This figure shows that there is a linear relation- 
ship between the module $\mathrm{I}_{\mathrm{sc}}$ and the operating temperature in case of the a-Si module corresponding to a short circuit temperature coefficient of about $0.09 \% /{ }^{0} \mathrm{C}$.

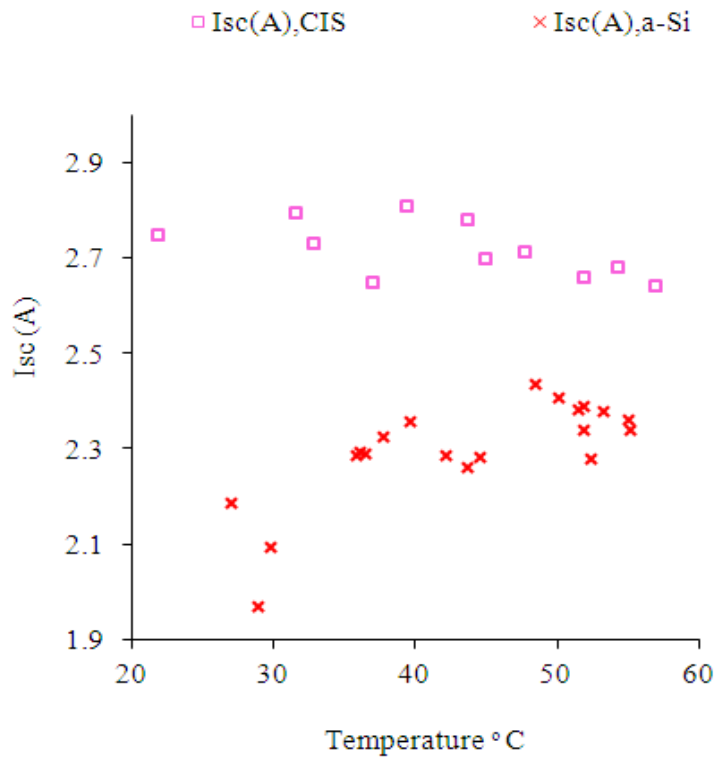

Figure 2. Variation of the short circuit current of the studied modules, as a function of temperature $T$

The detailed analysis of the generated currents from the CIS module has shown that at low and medium temperatures CIS current ranges at values close to the nominal with a positive short circuit current temperature coefficient $0.15 \% /{ }^{0} \mathrm{C}$. This coefficient is equal to the $\mathrm{I}_{\mathrm{sc}}$ temperature coefficient which can be calculated in Ref [13] for temperatures between 20 and $40{ }^{\circ} \mathrm{C}$. However over the whole range of working temperature this coefficient remains approximately constant with a slight tendency to decrease. A negative temperature coefficient of the short circuit current is indicated in ref [14].

The plots of the open circuit voltage, $V_{\text {oc }}$ Fig. 3, show that $\mathrm{V}_{\mathrm{oc}}$ decreases almost linearly when temperature increases. The influence of the temperature on $\mathrm{V}_{\mathrm{oc}}$ is greater for the CIS compared to that for the a-Si module ensuing to open circuit voltage temperature coefficients of around $-0.35 \% /{ }^{0} \mathrm{C}$ and $-0.32 \% /{ }^{0} \mathrm{C}$ respectively.

The variation of maximum power and efficiency as a function of temperature is depicted in Fig. 4. Maximum power in case of CIS decreases almost linearly when temperature increases, due to the linear drop in voltage.

It seems thus, that the small changes in the current do not substantially affect the power produced by the modules and the temperature coefficients of $\mathrm{P}_{\mathrm{m}}$ are approximately $-0.25 \% /{ }^{0} \mathrm{C}$ and $-0.45 \% /{ }^{0} \mathrm{C}$ for a-Si and CIS, respectively. $\mathrm{a}-\mathrm{Si}$ has the lowest power temperature coefficient which is in agreement to the calculated one in Ref [15] for a PV module of the same manufacturer. Power temperature coefficients for the CIS modules do not converge; however these modules are from different manufacturers.

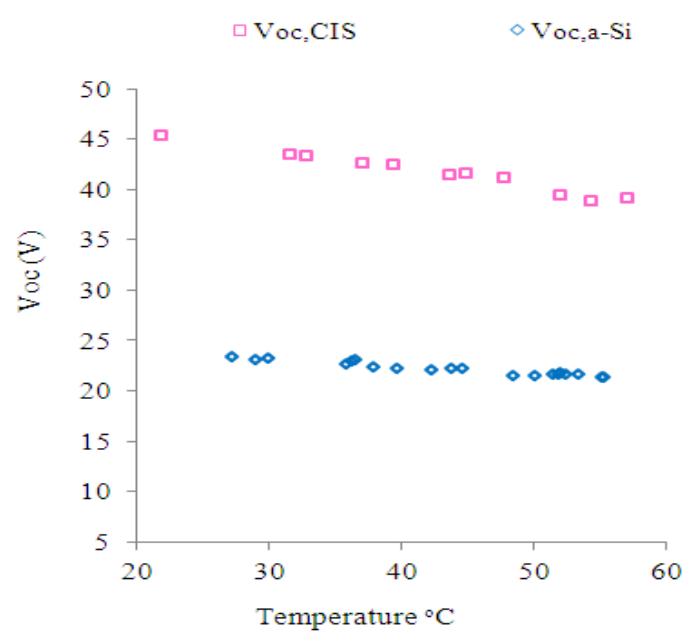

Figure 3. Open circuit voltage $V_{O C}$ of the tested modules as a function of temperature $T$

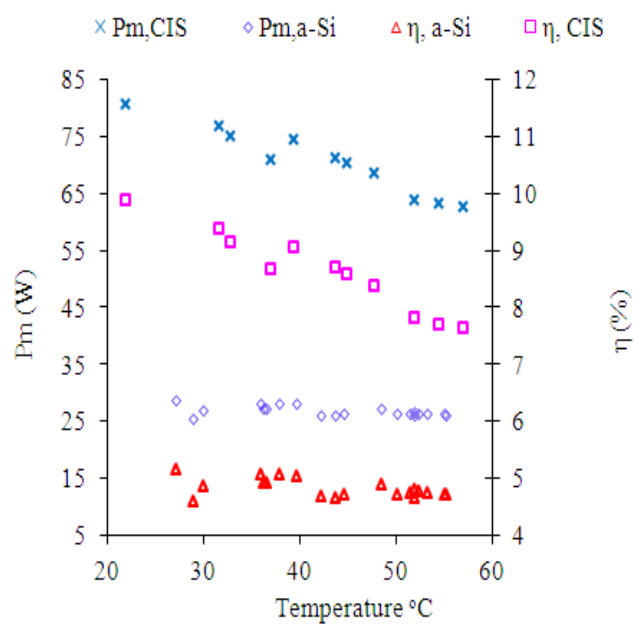

Figure 4. Power and efficiency graphs versus module temperature of the modules under investigation

The efficiency with regards to the temperature is mainly affected in the case of CIS, while it remains almost constant and near the rated value for a-Si. Our experimental results have shown that at low and medium temperatures $\left(25^{\circ} \mathrm{C}\right.$ to $45^{\circ} \mathrm{C}$ ) CIS efficiency ranges at values close to the nominal $(9.4 \%$ to $9 \%)$, while for higher temperatures decreases up to $7.6 \%$. A temperature coefficient of approximately $-0.6 \%$ / ${ }^{0} \mathrm{C}$ over the entire temperature range has been calculated. On the contrary, it is remarkable that the efficiency of a-Si is less dependent on temperature and remains within the nominal level, with a temperature coefficient equal to $-0.27 \% /{ }^{0} \mathrm{C}$.

The dependence of the fill factor on the temperature, for the modules under investigation, is presented in Fig. 5. This figure shows that there is a linear relationship between the averaged fill factor and the operating temperature resulting to equal temperature coefficients of approximately $0.21 \%$ / ${ }^{0} \mathrm{C}$. On the other hand the plots of their power coefficients 
$\mathrm{Pm} / \mathrm{Pstc}$, show that CIS is predominant against a-Si, despite its sharp decrease as a function of temperature.

Fig. 6 depicts the monthly averaged module temperatures and conversion efficiency for each module. The PV module efficiency is slightly reduced when module's temperature is high (from March to September) in case of the CIS module, whereas the a-Si module is not severely affected, due to its power dependence on temperature.

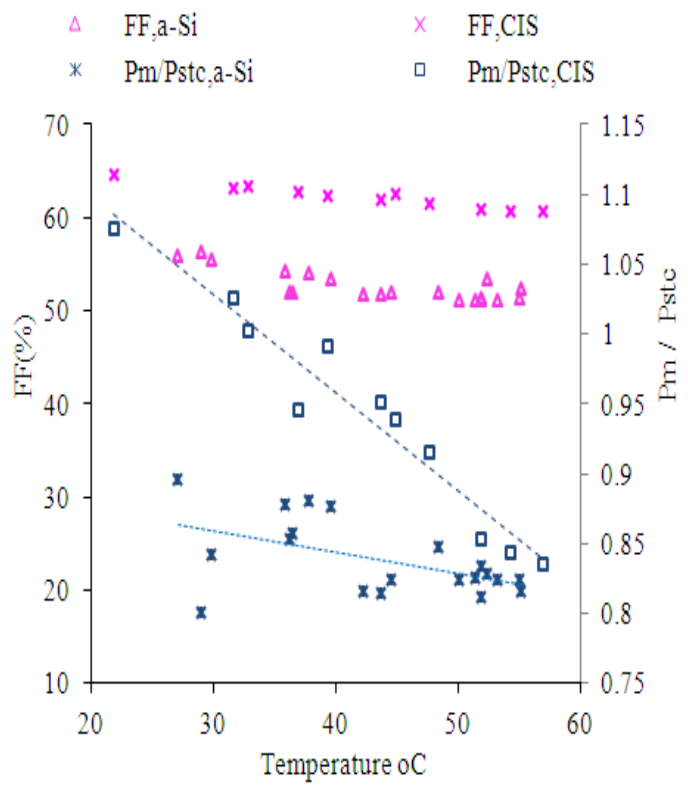

Figure 5. Fill factor and Pmax/Pstc as a function of temperature plotted for the modules a-Si and CIS

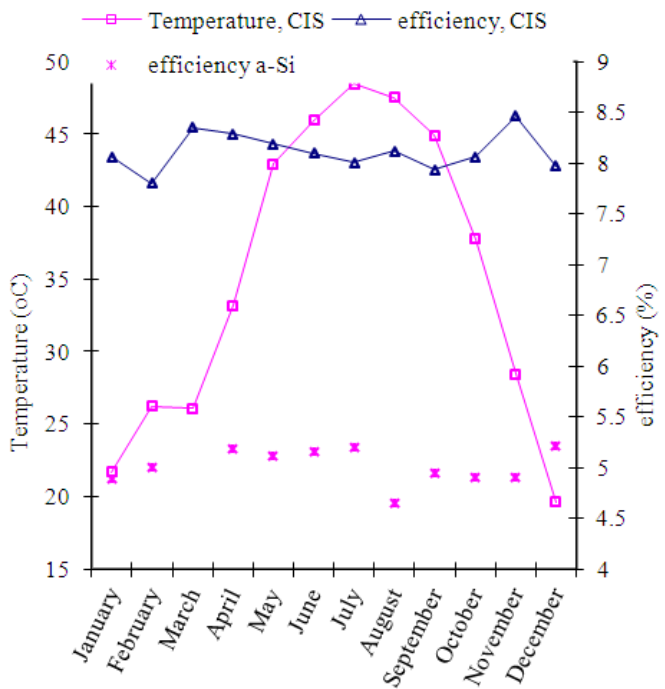

Figure 6. PV module temperature and conversion efficiency per month for a-Si and CIS module

The variations of series $R_{s}$ and parallel $R_{p}$ resistances of the a-Si and the CIS module versus the working temperatures are plotted in Fig. 7.

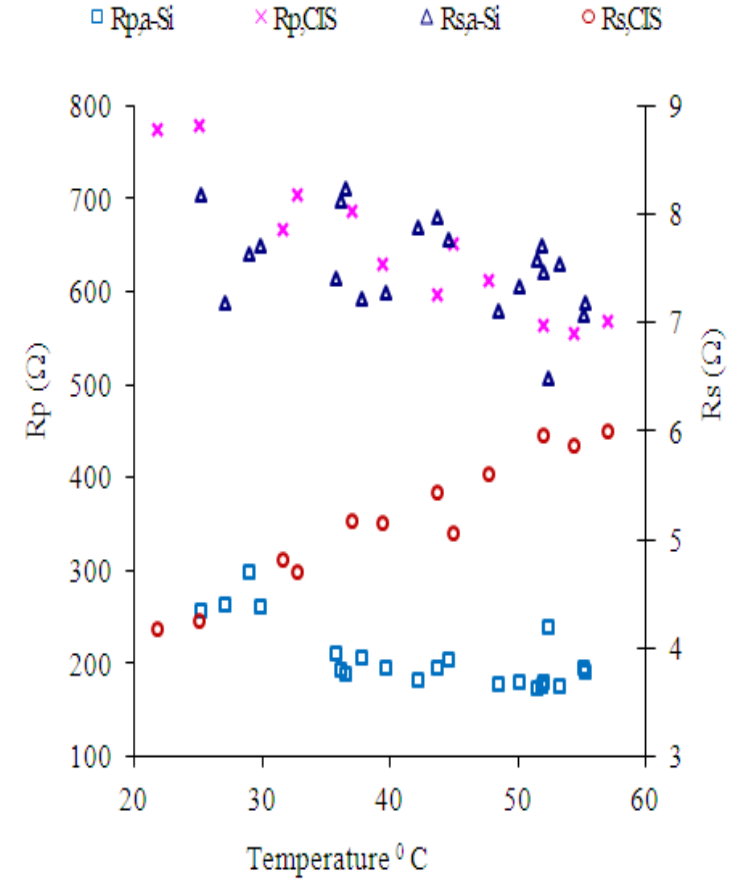

Figure 7. Variation of $R s$ and $R p$ with the working temperature of the studied modules

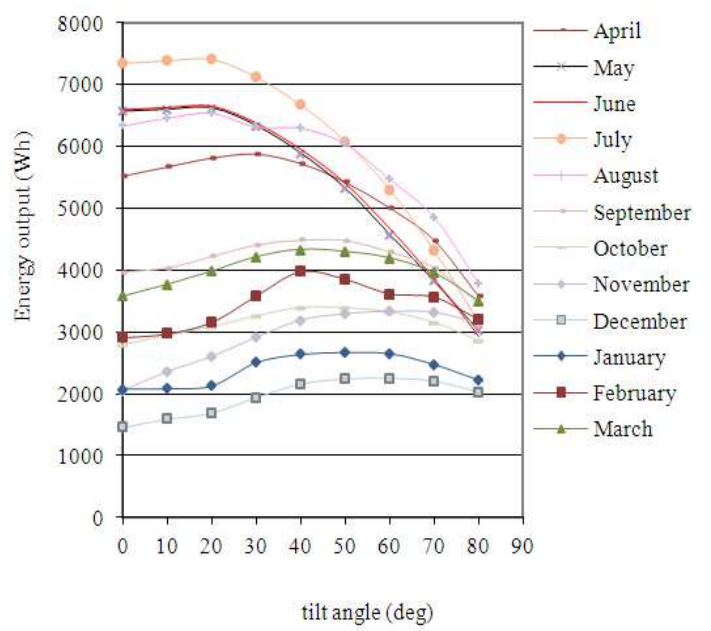

Figure 8. Energy yield (Wh) for the a-Si module as a function of the tilt angle during one year

These results indicate that for the a-Si module both of resistances decrease slightly with temperature. However for the CIS module, $R_{p}$ decreases and $R_{s}$, which adjusts the maximum power voltage, increases in a more intense way.

Tests performed at various tilt angles have investigated the impact of this angle on to the energy yield per month. The results depicted in Fig.8, show that the maximum energy produced, (and the solar radiation incident on the PV. module) corresponds to the modules tilt angles of about 20 degrees in the summer and 50 degrees in the winter. 
Table 1. Characteristic parameters of the PV modules concerning the type, rated power, $P_{M P P}$, open circuit voltage $V_{\text {oc }}$, short circuit current $I_{s c}$, voltage

\begin{tabular}{|c|c|c|c|c|c|c|}
\hline Manufacturer & $\mathbf{P}_{\text {MPP }}(\mathbf{W p})$ & $\operatorname{Voc}(V)$ & $\operatorname{Isc}(\mathrm{A})$ & $V_{M P P}(V)$ & $\mathbf{I}_{\text {MPP }}(\mathbf{A})$ & Efficiency (\%) \\
\hline a-Si module & 32 & 22.8 & 2.5 & 16.8 & 1.92 & 5.6 \\
\hline CIS module & $75+/-10 \%$ & 44.1 & 2.85 & 31.3 & 2.4 & 9.4 \\
\hline
\end{tabular}

\section{Conclusions}

It has been observed that the parameter which is more affected by the variation of temperature is open circuit voltage. The open circuit voltage $\mathrm{V}_{\mathrm{oc}}$, varies almost linearly with increasing temperature and the percentage reduction is greater for the CIS than for the a-Si module. In the case of a-Si the short circuit current $I_{s c}$, shows a slight increase with increasing temperature. The experimental results at low and medium operating module temperatures have shown that the generated from the CIS current ranges at values close to the nominal or apparent with a positive current temperature coefficient. For higher temperatures as well as for the whole range of working temperature this coefficient remains approximately constant with a slight tendency to decrease.

Maximum power in case of CIS decreases as a function of temperature almost linearly, due to the linear drop in voltage, which is not the case for the a-Si module. Furthermore the increase in temperature affects the CIS module efficiency in particular, showing that at low and medium temperatures the efficiency ranges in values close to its nominal, while for temperature higher than $50^{\circ} \mathrm{C}$ it is reduced enough. It is noteworthy that the increase in temperature does not affect the same the efficiency of a-Si, which remains very close to the rated value. The dependence of fill factor on temperature, for the modules under investigation shows linear decrease with increasing temperature and equal temperature coefficients. The annual behavior of PV modules indicates that the efficiency decreases with temperature only for the CIS, while for the a-Si module is not severely affected.

The series and parallel resistances, for the a-Si module decrease slightly with temperature, while for the CIS module the parallel decreases and the series resistance increases more sharply, affecting the short- circuit current.

Experiments at different tilt angles present the estimation of the generated energy per month in real / outdoor conditions.

\section{References}

[1] M C Alonso García, J.L. Balenzategui, "Estimation of photovoltaic module yearly temperature and performance based on nominal operation cell temperature calculations", Renewable Energy 29 (2004) pp.1997-2010
[2] V. Perraki, and V. Georgitsas, "Seasonal performance of monocrystalline silicon modules in a Mediterranean site", $25^{\text {th }}$ EUPVSEC and $5^{\text {th }}$ WCPVEC, Valencia 2010, pp 42814284

[3] Takuro Ihara, Hironori Nishihara, Fuji Electric review Vol. 49, No 2, pp 49-54

[4] K Akhmad, H Okamoto, F Yamamoto, A Kitamura, "Long term performance modelling of amorphous silicon photovoltaic module", Jpn. J. Appl. Phys. Part 1, Regular papers short

[5] A J Car, T L Pryor, "A comparison of the performance of different PV module types in temperature climates", Solar Energy 76 (2004), pp. 285-294

[6] Anatoli Chatzipanagi,Francesco Frontini, Sebastian Dittmann, Investigation of the influence of module working temperature on the performance of BIPV modules, $27^{\text {th }}$ EUPVSEC 2012,pp 4192-4197

[7] W Mieke, "Hot climate performance comparison between poly-crystalline and amorphous silicon cells connected to an utility mini-grid”. Proceedings of Solar 98, 36th Annual Conference of the Australian and New Zealand Solar Energy Society, Christchurch, New Zealand, 1998, pp. 464- 470.

[8] V.Perraki and G.Tsolkas "Evaluating PV System Performance of four different module technologies" $27^{\text {th }}$ EUPVSEC, Hamburg, 2012, pp 4236-4239.

[9] J Merten, J Andreu, "Clear separation of seasonal effects on the performance of amorphous silicon solar modules by outdoor I-V measurements" Solar Energy Materials \& Solar Cells, 52 (1998), pp11-25

[10] T Carlsson, "Experimental Setup of Full Scale Field Tests of CdTe and CIS Thin Film PV Modules", Master Thesis, Teknillinen Korkeakoulou, 2001

[11] Solveig Roschier, "Development of procedures for performance measurements and lifetime testing on thin film photovoltaic devices", Thesis, Helsinki University, Finland, 2002

[12] PV* SOL Expert 4.5, Valentin Energie Software

[13] Kandil M. Kandil, Majida S. Altouq, Asma M. Al-assad, Latifa M. Alshamari, Ibrahim M. Kadad, Adel A. Ghoneim, "Investigation of performance of CIS PV modules under different environmental conditions", Smart Grid and Renewable Energy, 2011, 2,375-387

[14] Matthew.T. Boyd, Douglas T. Reindl and Brian P. Dougherty,"Evaluation and validation of equivalent circuit photovoltaic solar cell performance models",Journal of Solar Energy Engineering,vol 133,2011. 
[15] G. Makridis, B. Zinsser, M. Norton and G. Georgiou, "Performance of photovoltaics under actual operating conditions", www.intechopen.com, Technology » "Third Generation

Photovoltaics", edited by V.Fthenakis, ISBN

978-953-51-0304-2, Published: March 16, 2012, pp 201-223. 